\title{
Posttransplant Lymphoproliferative Disorder involving the Larynx
}

\author{
${ }^{1}$ Nikita Kohli, ${ }^{2}$ Jared M Wasserman
}

\begin{abstract}
Aim: Our goal is to present an unusual case of posttransplant lymphoproliferative disorder (PTLD) involving the larynx. We aim to expand the literature regarding head and neck manifestations involving PTLD and for otolaryngologists to include PTLD in their differential diagnosis of laryngeal lesions in patients who have a history of solid organ transplantation.
\end{abstract}

Background: Posttransplant lymphoproliferative disorder occurs in immunocompromised patients following solid organ transplantation. Head and neck manifestations most commonly involve Waldeyer's ring with the larynx and trachea being relatively uncommon sites of disease. However, lesions of the larynx can cause acute airway obstruction and rare fatalities have been reported in the literature.

Case report: We present the case of a 51-year-old female with a history of renal transplantation, who presented to the office after an incidental supraglottic lesion was discovered during intubation for cochlear implant placement. A mucosalized lesion arising from the right aryepiglottic fold with dynamic obstruction of the airway was noted on office endoscopic examination. Imaging revealed extension of the mass into the right posterolateral hypopharynx. The patient was taken to the operating room for microdirect laryngoscopy and $\mathrm{CO}_{2}$ laser excision of the mass. The lesion was excised with negative gross margins. Final pathology revealed plasmacytoma-like PTLD and the patient was referred back to her transplant team for modulation of her immunosuppressive therapy.

Conclusion: We present the case of an incidental supraglottic lesion in a posttransplant patient. Special emphasis should be given to the tissues of Waldeyer's ring and larynx.

Clinical significance: Posttransplant lymphoproliferative disorder, while rare, should be kept in the differential diagnosis of pharyngeal and airway lesions in patients with a history of solid organ transplantation. Expansile lesions can cause acute airway obstruction. Urgent evaluation and treatment of theses lesions is necessary in these circumstances.

Keywords: Adult, Airway obstruction, Immunocompromised, Laryngology, Posttransplant lymphoproliferative disorder.

\footnotetext{
${ }^{1}$ Resident ${ }^{2}$ Private Practitioner

${ }^{1}$ SUNY Downstate Medical Center, Brooklyn, New York, USA

${ }^{2}$ The Voice and Swallowing Center ENT and Allergy Associates, Hackensack, New Jersey, USA
}

Corresponding Author: Nikita Kohli, Resident, SUNY Downstate Medical Center, Brooklyn, New York, USA Phone: 718-270-1638, e-mail: nikitavkohli@gmail.com
How to cite this article: Kohli N, Wasserman JM. Posttransplant Lymphoproliferative Disorder involving the Larynx. Int J Head Neck Surg 2016;7(1):53-56.

\section{Source of support: Nil}

\section{Conflict of interest: None}

\section{INTRODUCTION}

Posttransplant lymphoproliferative disorder (PTLD) was first reported in 1968 in renal transplant recipients. ${ }^{1}$ It occurs in immunocompromised patients following solid organ transplantation. The mechanism of the disease involves B lymphocyte proliferation in immunosuppressed individuals. Epstein Barr virus (EBV) has been implicated as an etiologic agent of the disease through infection of circulating B lymphocytes. Host response is subsequently generated by cytotoxic $T$ cells. These $\mathrm{T}$ cells subsequently cause the atypical lymphocytosis noted on pathologic specimens. ${ }^{2}$ While PTLD can occur in any organ system, the highest incidences of the disease occur after lung, heart, and intestinal transplants while kidney transplants have among the lowest rates of PTLD. ${ }^{3}$

In patients with head and neck manifestations, PTLD typically affects Waldeyer's ring. A 1990 review found that the tonsils and adenoids were among the most common sites of PTLD involving the head and neck. ${ }^{4}$ Less common lesions involve the oral cavity, larynx and trachea. ${ }^{3}$

Posttransplant lymphoproliferative disorder may be divided into different subtypes including plasma cell hyperplasia, polymorphic, and monomorphic forms, which is based on the World Health Organization (WHO) classification. ${ }^{5}$ Plasma cell hyperplasia is the least severe form of disease and may represent a pattern similar to infectious mononucleosis. The polymorphic form of the disease includes polyclonal and monoclonal forms and is characterized by distortion of plasma cell architecture and organ invasion. The monomorphic form of PTLD is the most aggressive phase of disease and can also be characterized as malignant lymphoma. The pathologic classification of these tumors may be used to predict patient response to immunosuppression. Patients with plasma cell hyperplasia and the polymorphic form of disease tend to be more likely to respond to reduction in the level of immunosuppression. ${ }^{6}$ 
Demographically, children are most commonly affected with a threefold increased chance of developing PTLD thought to be secondary to the higher incidence of EBV in the pediatric population. ${ }^{7}$ A retrospective case series by Lattyak et al estimated that approximately 10\% of pediatric patients undergoing liver transplantation will eventually develop PTLD in the head and neck. ${ }^{8}$ Patients with laryngeal or tracheal lesions may present with signs of acute airway obstruction, such as stridor, cough, and dyspnea. Other times, clinical findings may resemble the signs of infectious mononucleosis including fevers, malaise, odynophagia, and lymphadenopathy. On physical exam, hypertrophy of the tissues of Waldeyer's ring may be noted in addition to mucosal masses of the larynx and trachea on laryngoscopy.

A case series of children undergoing liver transplantation was reviewed at a Belgian hospital in a 2002 prospective study. ${ }^{9}$ In this study, 77 pediatric liver transplant recipients were followed for a 3-year period and monitored for the development of PTLD in the head and neck. Eight of these patients developed acute nonbacterial tonsillitis and those who were positive for EBV (as noted by positive IgM antibodies) underwent tonsillectomy. These patients also underwent concomitant reduction in the level of immunosuppression.

While the larynx and trachea appear to be less common sites of PTLD, multiple case reports of PTLD have been reported involving the upper airway. For example, a 6-year old boy with a history of liver transplantation presented with persistent stridor despite treatment for suspected viral laryngotracheitis. ${ }^{2}$ Following admission, the patient experienced acute airway obstruction and was taken to the operating room for emergent direct laryngoscopy, bronchoscopy and tracheostomy. On bronchoscopy, a 1 to $2 \mathrm{~cm}$ subglottic granulomatous mass was found causing over $90 \%$ airway obstruction.

One episode of sudden respiratory arrest and death secondary to PTLD was found in the literature. A 3-year old female on cytotoxic therapy following heart transplantation presented to the hospital with stridor and respiratory distress. Of note, she had developed hypertrophy of her tonsils and adenoids following transplantation and was scheduled to undergo elective tonsillectomy and adenoidectomy. On autopsy results, the patient was found to have hypertrophy of the tonsils, adenoids as well as the massive enlargement of the uvula to the level of the hyoid. It was speculated that hypertrophy of the tonsils and uvula contributed to significant upper airway narrowing and death. Posttransplant lymphoproliferative disorder was confirmed in these tissues and evidence of disseminated disease was found in the cervical lymph nodes, kidneys, and lungs. ${ }^{10}$
One report involved a 2-year old patient after liver transplantation who presented with stridor and was noted to have edema of the epiglottis. While laryngeal biopsy did not reveal the source of the lesion, PTLD was confirmed following tonsillectomy and adenoidectomy. ${ }^{11}$ Banks et al reported on a case of PTLD involving the larynx and trachea. The patient had a history of congenital nephrotic syndrome, bilateral nephrectomies, and subsequently underwent renal transplant at 10 months of age. ${ }^{3}$ The patient had previously presented with cervical lymphadenopathy that was positive for PTLD on open biopsy. Several years later, the patient presented to the emergency room with fevers, worsening cough, and shortness of breath with computed tomography (CT) chest revealing a paratracheal mass. The patient then underwent direct laryngoscopy, bronchoscopy, and biopsy of the tracheal mass as well as a soft tissue mass of the right laryngeal ventricle that was positive for PTLD. The patient's lesions eventually resolved following the reduction of immunosuppressive therapy and initiation of chemotherapy. ${ }^{3}$

Lesions of the oral cavity, while rare, have also been reported in the literature. A 2008 case series reported 3 cases of PTLD in the oral cavity following organ transplantation. ${ }^{6}$ These lesions involved the oral tongue, gingiva and buccal vestibule. These lesions involved the buccal mucosa and gingiva and were treated with wide local excision and modification of immunosuppression.

While no randomized control trials or guidelines have been published to delineate the standards of care for management of PTLD, treatment of the disease involves reduction of the immunosuppressive agent by up to $50 \%$. However, there is a risk of graft rejection in these cases thus monitoring of graft function is necessary. Further, patients require close monitoring for clinical deterioration and weekly EBV evaluation may be performed to monitor viral load. ${ }^{6}$ Cases refractory to this treatment may require antivirals, chemotherapy, immunotherapy, or definitive surgical resection of the offending lesion. In some cases, positron emission tomography and computed tomography (PET-CT) may be used for posttreatment surveillance to evaluate for resolution of the disease. ${ }^{3}$

\section{CASE REPORT}

The patient is a 51-year-old woman with a past medical history of renal failure and renal transplant 18 years prior to presentation. She was referred for laryngology evaluation after attempted intubation for elective cochlear implantation secondary to profound sensorineural hearing loss. During laryngoscopy, intubation was aborted when the anesthesiologist encountered an obstructing supraglottic mass. 
The patient denied any shortness of breath or difficulty swallowing. She had a normal voice, no stridor, and denied globus sensation. Flexible laryngoscopic examination revealed a solid appearing, smoothly mucosalized mass involving the right aryepiglottic fold (Fig. 1). This mass appeared to be dynamically obstructing the airway. However, the glottic and subglottic airway was normal. Computed tomography scan of the neck with intravenous contrast showed an enhancing, solid mass, which was contiguous with the right aryepiglottic fold. There were no cystic components seen and the lesion protruded into the right posterolateral hypopharyx (Figs 2 and 3).

The patient was taken to the operating for microlaryngoscopy with $\mathrm{CO}_{2}$ laser resection of the mass. After communicating with the anesthesiologist, the patient was safely intubated. The lesion was resected with appropriate gross margins. After removal, the airway was clear and widely patent. The patient was extubated without difficulty. Pathology revealed plasma cells consistent with Plasmacytoma-like PTLD (Figs 4A and B). The

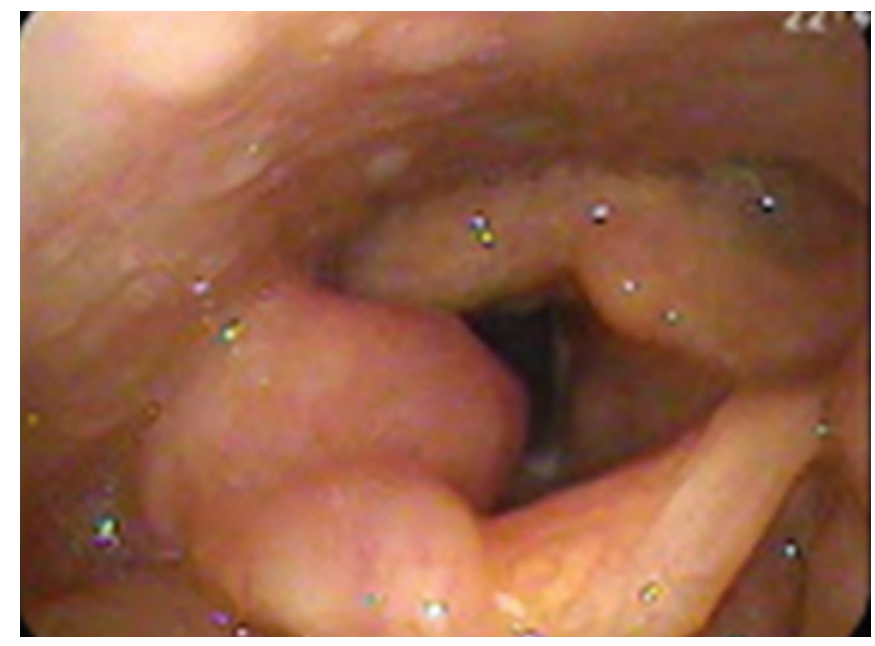

Fig. 1: Supraglottic mass on fiberoptic exam involving right aryepiglottic fold

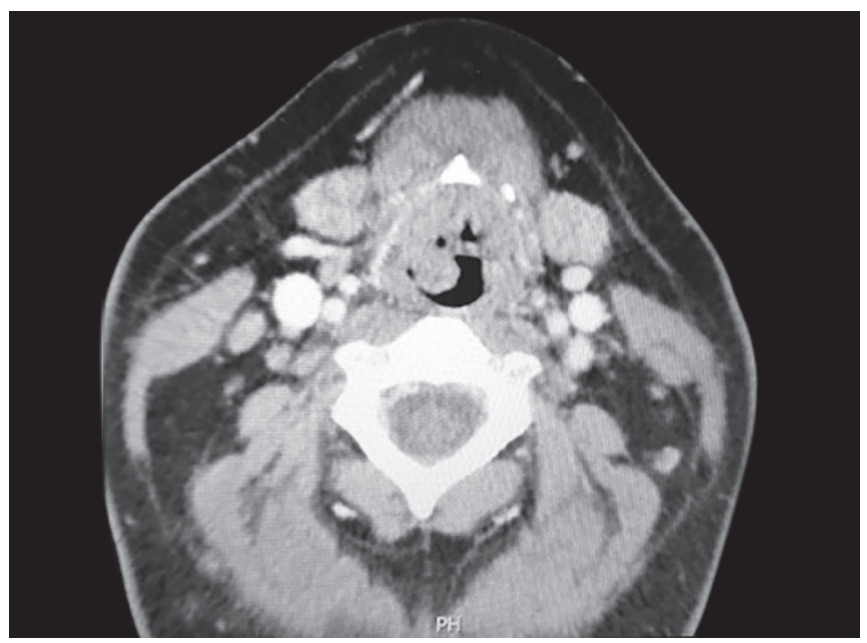

Fig. 2: Computed tomography neck revealing superior aspect of mass arising from right aryepiglottic fold patient did well postoperatively and was referred back to her transplant team for reduction of her immunosuppressive regimen.

\section{DISCUSSION}

The case above demonstrates an incidental lesion of PTLD involving the supraglottic larynx. While the larynx is an uncommon site for PTLD, multiple case reports of PTLD involving the upper airway have been reported. For patient with a history of solid organ transplantation and presenting symptoms consistent with upper airway obstruction, there must be a high index of suspicion for PTLD. Tissue biopsy is required for the diagnosis. Treatment typically involves reduction of the immunosuppressive agent as well as chemotherapy for non-responders. Antivirals including acyclovir have been used in certain patients as well, which are designed to target EBV.

\section{CONCLUSION}

We present on a relatively rare instance of PTLD involving the supraglottic larynx in a patient who underwent bilateral renal transplantation. Posttransplant lymphoproliferative disorder and its head and neck manifestations should be kept in mind when evaluating a patient with history of solid organ transplantation. In particular, additional emphasis on the tissues of Waldeyer's ring as well as the larynx should be placed to assess for tissue hypertrophy or masses. Surgical biopsy of suspicious or symptomatic lesions should be performed.

\section{CLINICAL SIGNIFICANCE}

In this case report, we report on a relatively rare occurrence of PTLD involving the larynx. While this lesion was incidentally discovered, other cases of PTLD involving the larynx and trachea and may be found in patients

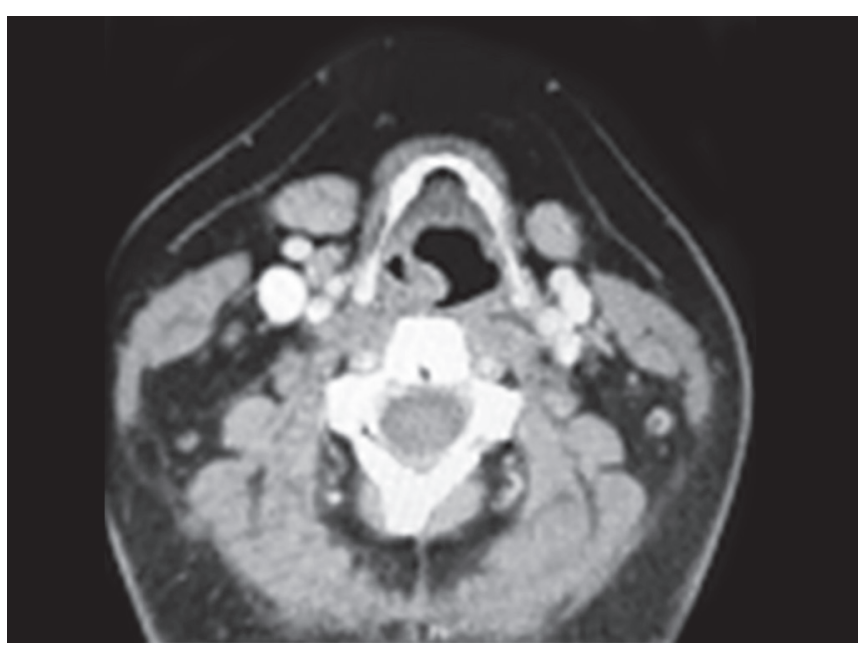

Fig. 3: Extension of mass into right posterolateral hypopharynx on CT neck 


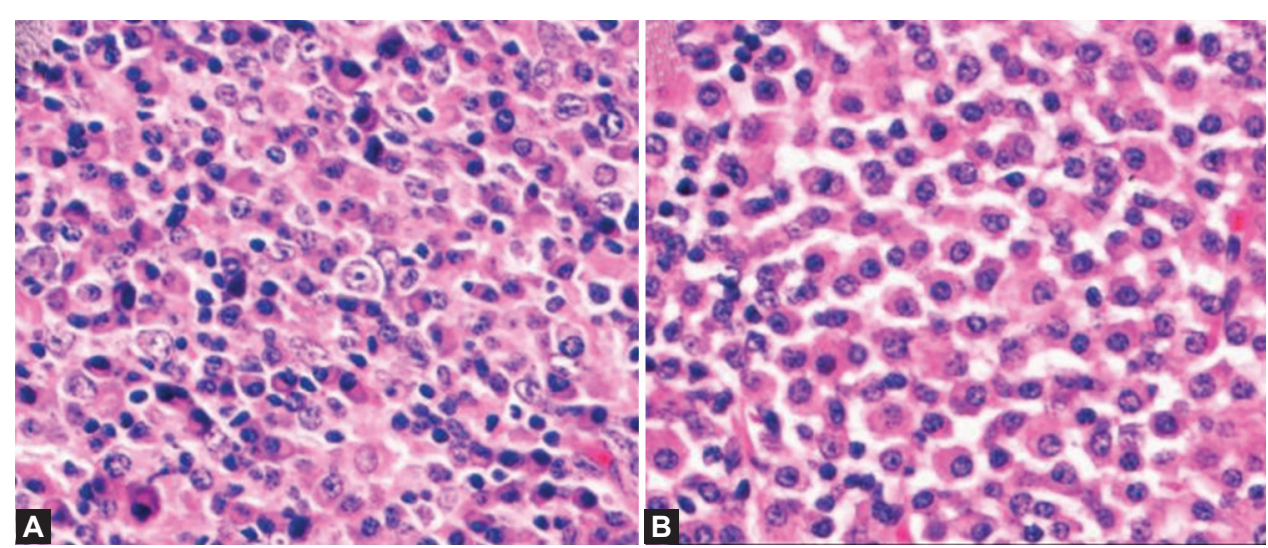

Figs 4A and B: Representative image of pathology for plasmacytoma-like PTLD: (A) numerous plasma cells with occasional admixed histiocytes and immunoblasts; and (B) sheets of well-differentiated plasma cells ${ }^{12}$

presenting with signs and symptoms of upper airway obstruction. Otolaryngologists should maintain PTLD in their differential diagnosis when managing patients who have a history of organ transplantation. Multiple case reports have highlighted the fact that although rare, PTLD of the larynx and trachea can cause lifethreatening airway obstruction. For children developing new onset sleep apnea with adenotonsillar hypertrophy after solid organ transplantation, prompt tonsillectomy and adenoidectomy should be considered as a primary form of management as severe hypertrophy of these tissues may cause acute, life-threatening airway obstruction. ${ }^{10}$ The primary role of the otolaryngologist remains for management of the airway, biopsy and debulking of obstructive lesions.

Following surgical management, reduction of immunosuppression is necessary, which requires coordination with the oncologist and transplant team. The addition of antiviral therapy may also be necessary to reduce the EBV viral load. Thus, close communication and coordination is necessary between the otolaryngologist, oncologist, and transplant physicians for optimal management.

\section{REFERENCES}

1. Murray JE, Wilson RE, Tilney NL, Merrill JP, Cooper WC, Birtch AG, Carpenter CB, Hager EB, Dammin GJ, Harrison JH. Five years exprience with renal transplantation with immunosuppressive drugs: survival, function, complications and the role of lymphocyte depletion by thoracic duct fistula. Ann Surg 1968 Oct;168(3):416-435.

2. Rafferty MA, Devaney D, Russell J. Case report: an unusual cause of stridor in a post liver transplant 6-year old. Int J Pediatr Otorhinolaryngol 2000 Aug;54(2-3):149-151.
3. Banks CA, Meier JD, Stallworth CR, White DR. Recurrent posttransplant lymphoporliferative disorder involving the larynx and trachea: case report and review of the literature. Ann Otol Rhinol Laryngol 2012 May;121(5):291-295.

4. Sculerati N, Arriaga M. Otolaryngologic management of posttransplant lymphoproliferative disease in children. Ann Otol Rhinol Laryngol 1990 Jun;99(6 pt 1):445-450.

5. Harris NL, Ferry JA, Swerdlow SH. Posttransplant lymphoproliferative disorders: summary of society for hematopathology workshop. Semin Diagn Pathol 1997 Feb;14(1):8-14.

6. Ojha J, Islam N, Cohen DM, Marshal D, Reavis MR, Bhattacharyya I. Posttransplant lymphoproliferative disorders of oral cavity. Oral Surg Oral Med Oral Pathol Oral Radiol Endod 2008 May;105(5):589-596.

7. Jain A,NalesnikM,Reyes J,PokharnaR,MazariegosG,GreenM, Eghtesad B, Marsh W, Cacciarelli T, Fontes P, Abu-Elmagd K, et al. Posttransplant lymphoproliferative disorders in liver transplantation: a 20-year experience. Ann Surg 2002 Oct; 236(4):429-437.

8. Lattyak BV, Rosenthal P, Mudge C, Roberts JP, Renze JF, Osorio RW, Emond JC, Lalwani AK. Posttransplant lymphoproliferative disorder presenting in the head and neck. Laryngoscope 1998 Aug;108(1):1195-1198.

9. Nouwen J, Smets F, Hamoir M, Rombaux P, Sokal EM. Acute tonsillitis as the first manifestation of posttransplant lymphoproliferative disorder. Ann Otol Rhinol Laryngol 2002 Feb;111(2):165-168.

10. Hague K, Catalano P, Strauchen J, Rothschild M, Fyfe B. Posttransplant lymphoproliferative disorder presenting as sudden respiratory arrest in a 3-year-old child. Ann Otol, Rhinol Laryngol 1997 Mar;106(3):244-247.

11. Rosbe KW, Perez-Atay de AR, Kenna M. Pathology forum: quiz case 1 . Diagnosis: posttransplant lymphorproliferative disease (PTLD) of the epiglottis. Arch Otolaryngol Head Neck Surg 2000 Sep;126(9):1157-1158.

12. Richendollar BG, HE, Cook JR. Extramedullary plasmacytomalike posttransplantation lymphoproliferative disorders: clinical and pathologic features. Am J Clin Pathol 2009;132(4):581-588. 